\title{
11 Genetic Statistical Model to Estimate Epistasis, Additive and Dominance Genetic Effects Using Advanced Populations
}

\author{
A. El. A. Khalaf*, T. M. Sabet*, A. A. M. Yassein* \\ Agronomy Dept. and * Genetics Dept.,Plant Breeding Section, Faculty of Agriculture, \\ Fayoum Univ., 6351-Fayoum, Egypt.
}

Received: $15 / 1 / 2017$

Accepted: 8/5/2017

\section{INTRODUCTION}

Several designs have been developed for genetic analysis of metric traits using the earlier six population means ( i. e., $\overline{\mathrm{P}}_{1}, \overline{\mathrm{P}}_{2}, \overline{\mathrm{F}}_{1}, \overline{\mathrm{F}}_{2}, \overline{\mathrm{BC}}_{11}$ and $\overline{\mathrm{BC}}_{12}$ ). M a the r,
STIMATING gene effects contributing to metric traits has been investigated

in several studies. Most estimation methods focused on means of earlier filial $\left(\bar{F}_{1 \text { and }} \bar{F}_{2}\right)$ and backcross populations $\left(\overline{B C}_{11}\right.$ and $\left.\overline{B C}_{12}\right)$. In this paper, a novel approach is proposed to estimate gene effect using the data generated from advanced filial $\left(\bar{F}_{3}, \bar{F}_{4} \ldots, \bar{F}_{n}\right)$ and backcross populations $\left(\overline{B C}_{n 1}\right.$ and $\left.\overline{B C}_{n 2}\right)$. Where the following set of equations is derived by algebraic solution of $\bar{P}_{1}, \bar{P}_{2}, \bar{F}_{3}, \bar{F}_{4}, \overline{B C}_{31}$ and $\overline{B C}_{32}$ means:

Mean $(\mathrm{M})=\frac{1}{98}\left(49 \overline{\mathrm{P}}_{1}+49 \overline{\mathrm{P}}_{2}-64 \overline{\mathrm{BC}}_{31}-64 \overline{\mathrm{BC}}_{32}+128 \overline{\mathrm{F}}_{4}\right)$

Additive effect $(\mathrm{D})=\frac{1}{2}\left(\overline{\mathbf{P}}_{1}-\overline{\mathbf{P}}_{2}\right)$,

Dominance effect $(\mathrm{H})$

$=\frac{1}{49}\left(384 \overline{\mathrm{BC}}_{31}+384 \overline{\mathrm{BC}}_{32}-196 \overline{\mathrm{F}}_{3}-294 \overline{\mathrm{P}}_{1}-294 \overline{\mathrm{P}}_{2}+16 \overline{\mathrm{F}}_{4}\right)$,

Three types of epistasis:

Additive $\mathrm{x}$ additive effect $(\mathrm{I})=\frac{32}{49}\left(\overline{\mathrm{BC}}_{31}+\overline{\mathrm{BC}}_{32}-2 \overline{\mathrm{F}}_{4}\right)$,

Additive $\mathrm{x}$ dominance $\operatorname{effect}(\mathrm{J})=$

$\frac{1}{7}\left(32 \overline{\mathrm{BC}}_{31}-32 \overline{\mathrm{BC}}_{32}-28 \overline{\mathrm{P}}_{1}+28 \overline{\mathrm{P}}_{2}\right)$ and

Dominance $\mathrm{x}$ dominance effect $(\mathrm{L})=$

$\frac{16}{49}\left(98 \overline{\mathrm{F}}_{3}-68 \overline{\mathrm{F}}_{4}+49 \overline{\mathrm{P}}_{1}+49 \overline{\mathrm{P}}_{2}-64 \overline{\mathrm{BC}}_{31}-64 \overline{\mathrm{BC}}_{32}\right)$

The proposed equations have been proved by means of algebra, in addition to mathematical proof using real data. The results suggest that, the model can serve as a viable particularly to estimate epistasis, additive and dominance genetic effects using the data generated from advanced filial and backcross populations $\bar{P}_{1}, \bar{P}_{2}, \bar{F}_{3}, \bar{F}_{4}, \overline{B C}_{31}$ and $\overline{B C}_{32}$

Keywords: Advanced filial and backcross populations, Algebraic and Mathematical proof, Gene effects.

(1949) defined the scaling test for adequacy of the additive dominance model based on generation means of these six populations. Afterward, an improved model of this test have been formulated in different studies, where the by joint scaling 
test based on weighted least squares estimates of the same populations was proposed (Cavalli, 1952; Anderson \& Kempthorne, 1954; Jinks, 1956 and Hayman, 1957). Designs have been extended to estimate the different types of gene effects by analyzing means of some or all the six earlier populations. Accordingly six population's designs for estimating all types of gene effects have been proposed by Andrson \& Kempthorne (1954), Cockerham (1954) and Hayman (1958). However, model based on only varieties and their crosses have been suggested by Gardner \& Eberhart (1966) to estimate these gene effects.

In comparison, models based only on inbred lines have been adopted but are merely satisfactory for estimating epistatic effects. Kearsey \& Jinks (1968) suggested the triple test-cross of inbred lines to estimate the epistatic variation while Jinks et al. (1969) derived a simplified test for this purpose. Similarly, only backcrosses have been utilized in designs for testing epistasis. Mather \& Jinks (1971) and Chahal \& Jinks (1978) proposed standard backcross scale for testing epistasis in case of absence of inbred testers. The only design based on an advanced population was that proposed by Jinks \&Jones (1958). They postulated a five population design including $\overline{\mathrm{P}}_{1}, \overline{\mathrm{P}}_{2}, \overline{\mathrm{F}}_{1}, \overline{\mathrm{F}}_{2}$ and $\left.\overline{\mathrm{F}}_{3}\right)$ in case backcross progenies are not available. The defect of this design is that it is limited for estimating only single gene effects. Such classical designs are impractical for full analysis of metric traits if any of the earlier six populations is not available or its size is not quite enough for satisfactory analysis. On the other hand, in practice, breeders may have different advanced filial and backcross populations which are sufficient for perfect investigation. Generation mean analysis (Mather \& Jinks, 1982) consists of six populations, i.e. parent $1\left(\overline{\mathrm{P}}_{1}\right)$, parent $2\left(\overline{\mathrm{P}}_{2}\right)$, first filial $\left(\overline{\mathrm{F}}_{1}\right)$, second generation $\left(\overline{\mathrm{F}}_{2}\right)$, first filial of the backcross with recurrent parent $1\left(\overline{\mathrm{BC}}_{11}\right)$ and first filial of the backcross with recurrent parent $2\left(\overline{\mathrm{BC}}_{12}\right)$. Generation mean analysis is a useful technique that provides the estimation of the average effects of the genes (additive, dominance and epistasis) involved in the expression of quantitative traits such as yield and yield components which benefits breeders in designing the most appropriate breeding approaches for developing a new varieties (Pooni \& Treharne, 1994; Iqbal \& Nadeem, 2003; Checa et al., 2006 and Sharmila et al.,2007). Genome scans for quantitative trait loci (QTL) have proved to be a successful strategy for identifying genetic effects and interactions (Carlborg et al., 2004).

The present study proposed a design for estimating all types of gene effects based on means of advanced filial and backcross generation's means $\left(\overline{\mathrm{P}}_{1}, \overline{\mathrm{P}}_{2}, \overline{\mathrm{F}}_{4}, \overline{\mathrm{F}}_{3}, \overline{\mathrm{BC}}_{31}\right.$ and $\left.\overline{\mathrm{BC}}_{32}\right)$.

\section{PROTOCOL OF DEDUCTION}

The protocol employed for finding out the six formulas involves the following three methods:

1.Algebraic solution: The key point in this analysis is solution of means of the two filial generations to find values of each of the unknowns $\mathrm{H}$ and $\mathrm{L}$ in terms of population means. Meanwhile, D is considered as half of the difference between the two parents. These three derived parameters were used in compensation in specific appropriate group of populations to each of the gene effects.

2.Algebraic proof: Components of the population means that are involved in each formula are compensated in terms of gene effects.

3.Mathematical proof via working example: For this purpose, real data was utilized to obtain the six gene effects, which were used for calculating the expected genetic values according to their genetic components. Thereafter, these numerical means were used for re-estimating gene effects according to the deduced formulas.

$\bar{P}_{1}=$ mean of the superior parent or homozygous dominant, selfed.

$\bar{P}_{2}=$ mean of the inferior parent or homozygous recessive, selfed.

$\bar{F}_{3}=$ mean of the $3^{\text {rd }}$ selfing filial generation.

$\bar{F}_{4}=$ mean of the $4^{\text {th }}$ selfing filial generation.

$\overline{B C}_{31}=$ mean of the $3^{\text {rd }}$ backcross to

the larger parent generation (where

$\overline{B C}_{31}=\overline{B C}_{21} x \bar{P}_{1}$ and $\overline{B C}_{21}=\overline{B C}_{11} * \bar{P}_{1}$ and $\overline{B C}_{11}=\bar{F}_{1} * \bar{P}_{1}$

$\overline{B C}_{32}=$ mean of the $3^{\text {rd }}$ backcross to the smaller parent generation

where $\overline{B C}_{32}=\overline{B C}_{22} * \bar{P}_{2}$ and $\overline{B C}_{22}=\overline{B C}_{12} * \bar{P}_{2}$ and $\overline{B C}_{12}=\bar{F}_{1} * \bar{P}_{2}$

As shown in Fig. 1. 


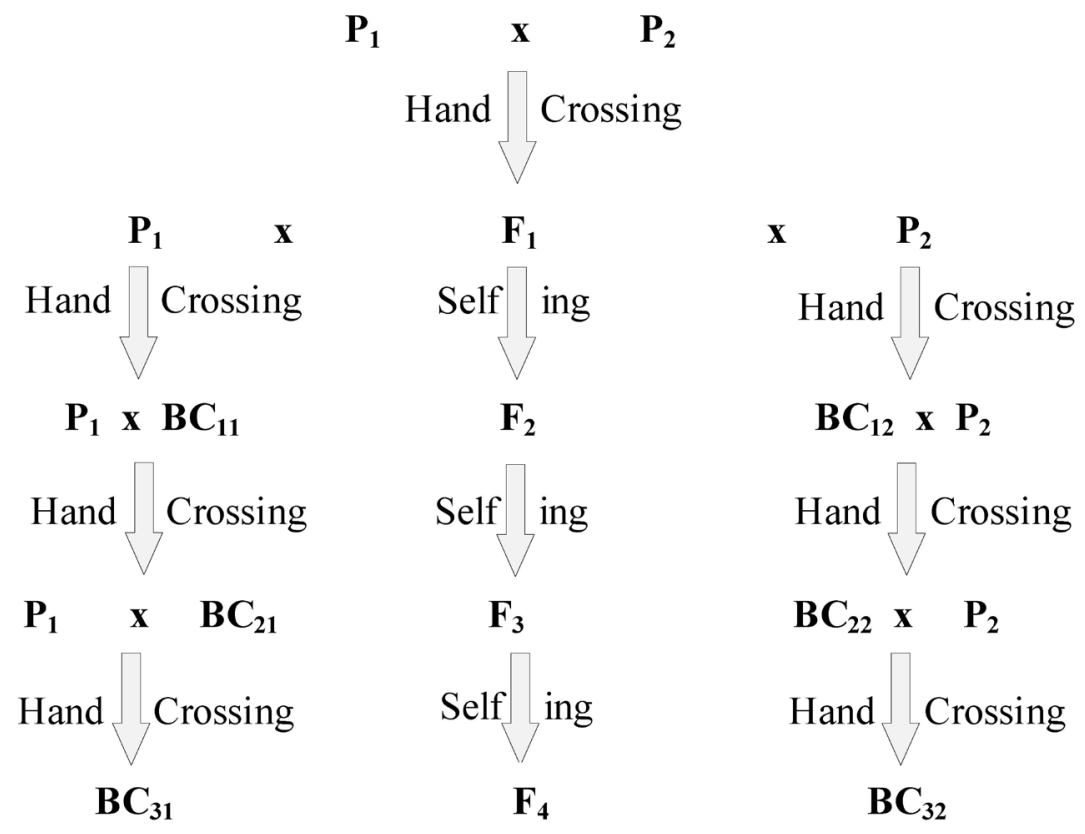

Fig. 1. Crossing chart indicating how the plant material was obtaine.

\section{Algebraic proof}

Components of population genetic means in case of adequacy of the additive-dominance model (Table 1):

$\overline{\mathrm{P}}_{1}=\mathrm{m}+\mathrm{d}$

$\overline{\mathrm{P}}_{2}=\mathrm{m}-\mathrm{d}$

$\bar{F}_{3}=m+1 / 4 h$

$\overline{\mathrm{F}}_{4}=\mathrm{m}+1 / 6 \mathrm{~h}$

$\overline{B C}_{31}=m+7 / 8 d+1 / 8 h$

$\overline{B C}_{32}=\mathrm{m}-7 / 8 \mathrm{~d}+1 / 8 \mathrm{~h}$

Components of population genetic means if non-allelic gene actions are involved (Table 1):

$$
\begin{aligned}
& \overline{\mathrm{P}}_{1}=\mathrm{m}+\mathrm{d} \\
& \overline{\mathrm{P}}_{2}=\mathrm{m}-\mathrm{d} \\
& \overline{\mathrm{F}}_{3}=\mathrm{m}+1 / 4 \mathrm{~h}+1 / 161
\end{aligned}
$$

$\overline{\mathrm{F}}_{4}=\mathrm{m}+1 / 8 \mathrm{~h}+1 / 641$

$\overline{\mathrm{BC}}_{31}=\mathrm{m}+7 / 8 \mathrm{~d}+1 / 6 \mathrm{~h}+49 / 64 \mathrm{i}+7 / 64 \mathrm{j}+1 / 641$

$\overline{B C}_{32}=m-7 / 8 d+1 / 6 h+49 / 64 i-7 / 64 j+1 / 641$

where (m) is mean, (d) is additive effect, (h) is dominance effect, , (i) is additive $\mathrm{x}$ additive effect, $(j)$ is additive $x$ dominance effect and (l) is dominance $\mathrm{x}$ dominance effect.

\section{Scaling test formula}

The scaling test A, B, C, D and E for additivedominance model were applied to test the appropriate genetic model formulae (A, B, C, D and $\mathrm{E}$ ) and their variances to test the presence of non- allelic gene interaction. If the value of A, B, $\mathrm{C}, \mathrm{D}$ and $\mathrm{E}$ equal about "zero" or insignificant, this means that epistasis (non - allelic interaction) is absent, so the simple genetic model (m), (d) and (h) is adequate. If any one of A, B, C, D and E was significantly different from" zero" this means the presence of non-allelic interaction and the scaling test analysis is inadequate and needs other scales or data transformation. The analysis should be proceeded to estimate the interaction types of gene effect involved, using the six-parameters genetic model. 
TABLE1. Coefficients of the parameters used in the genetic model for generation means analysis.

\begin{tabular}{|c|c|c|c|c|c|c|}
\hline \multirow{2}{*}{ Generations } & \multicolumn{6}{|c|}{ Generation Parameters } \\
\cline { 2 - 7 } & \multicolumn{3}{|c|}{ Simple Effect } & \multicolumn{3}{c|}{ Epistasis } \\
\cline { 2 - 7 } & $\mathbf{m}$ & $\mathbf{d}$ & $\mathbf{h}$ & $\mathbf{i}$ & $\mathbf{j}$ & $\mathbf{I}$ \\
\hline $\mathbf{P}_{\mathbf{1}}$ & 1 & +1 & 0 & +1 & 0 & 0 \\
\hline $\mathbf{P}_{\mathbf{2}}$ & 1 & -1 & 0 & +1 & 0 & 0 \\
\hline $\mathbf{F}_{\mathbf{3}}$ & 1 & 0 & $+1 / 4$ & 0 & 0 & $+1 / 16$ \\
\hline $\mathbf{F}_{\mathbf{4}}$ & 1 & 0 & $+1 / 8$ & 0 & 0 & $+1 / 64$ \\
\hline $\mathbf{B C}_{\mathbf{3 1}}$ & 1 & $+7 / 8$ & $+1 / 8$ & $+49 / 64$ & $+7 / 64$ & $+1 / 64$ \\
\hline $\mathbf{B C}_{\mathbf{3 2}}$ & 1 & $-7 / 8$ & $+1 / 8$ & $+49 / 64$ & $-7 / 64$ & $+1 / 64$ \\
\hline
\end{tabular}

Where: $(\mathrm{m}=$ mean effects; $[\mathrm{d}]=$ additive; $[\mathrm{h}]=$ dominance; $[\mathrm{i}]=$ additive $\mathrm{x}$ additive; $[\mathrm{j}]=$ additive $\mathrm{x}$ dominance; $[\mathrm{l}]=$ dominance $\mathrm{x}$ dominance)

$$
\begin{aligned}
& A=4 \bar{F}_{4}-2 \bar{F}_{3}-\bar{P}_{1}-\bar{P}_{2} \\
& B=2 \bar{F}_{4}-\overline{B C}_{31}-\overline{B C}_{32} \\
& C=32 \overline{B C}_{31}-32 \overline{B C}_{32}-28 \overline{\mathrm{P}}_{1}+28 \overline{\mathrm{P}}_{2} \\
& \mathrm{D}=98 \overline{\mathrm{F}}_{3}-68 \overline{\mathrm{F}}_{4}+49 \overline{\mathrm{P}}_{1}+49 \overline{\mathrm{P}}_{2}-64 \overline{B C}_{31}-64 \overline{B C}_{32} \\
& E=\overline{B C}_{31}+\overline{B C}_{32}-2 \overline{\mathrm{F}}_{4}
\end{aligned}
$$

Algebraic solution to deduce six parameters $M$, $D, H, I, J$ and $L$ :

The key point in this analysis is the solution of means of the $\overline{\mathrm{F}}_{3}$ and $\overline{\mathrm{F}}_{4}$ filial generations to find values of each of the unknown $H$ and $L$ in terms of population means as follow:

$\overline{\mathrm{F}}_{3}=\mathrm{m}+1 / 4 \mathrm{~h}+1 / 16 \mathrm{l}$

Multiplying to sides of formula by $1 / 4$, which can be rewritten as

$$
\begin{aligned}
& 1 / 4 \overline{\mathrm{F}}_{3}=1 / 4 \mathrm{~m}+(1 / 4 * 1 / 4) \mathrm{h}+(1 / 4 * 1 / 16) \mathrm{l} \\
& 1 / 4 \overline{\mathrm{F}}_{3}=1 / 4 \mathrm{~m}+1 / 16 \mathrm{~h}+1 / 64 \mathrm{l}
\end{aligned}
$$

Then subtract above formula from below one to get value of $h$

$$
\overline{\mathrm{F}}_{4}=\mathrm{m}+1 / 8 \mathrm{~h}+1 / 641
$$

$\overline{\mathrm{F}}_{4}-1 / 4 \overline{\mathrm{F}}_{3}=\mathrm{m}-1 / 4 \mathrm{~m}+1 / 3 \mathrm{~h}-1 / 16 \mathrm{~h}+1 / 64 \mathrm{l}-1 / 64 \mathrm{l}$

$$
\mathrm{h}=16 \overline{\mathrm{F}}_{4}-4 \overline{\mathrm{F}}_{3}-12 \mathrm{~m}
$$

When $\mathrm{h}$ is compensated in next formula

Egypt.J.Agron. Vol.39, No.1 (2017)

$$
\begin{aligned}
& \overline{\mathrm{F}}_{3}=\mathrm{m}+1 / 4 \mathrm{~h}+1 / 16 \mathrm{l} \\
& \text { We can estimate } \mathrm{L} \\
& \overline{\mathrm{F}}_{3}=\mathrm{m}+1 / 4\left(16 \overline{\mathrm{F}}_{4}-4 \overline{\mathrm{F}}_{3}-12 \mathrm{~m}\right)+1 / 16 \mathrm{l} \\
& \mathrm{L}=32 \overline{\mathrm{F}}_{3}-64 \overline{\mathrm{F}}_{4}+32 \mathrm{~m}
\end{aligned}
$$

......Equation (2)

For estimating $\mathbf{M}$ by summing $\bar{P}_{1}$ and $\bar{P}_{2}$ means and substitution from sum of $\overline{B C}_{31}$ and $\overline{B C}_{\mathrm{a2}}$ as follow:

$$
=\left(\bar{P}_{1}+\bar{P}_{2}\right)-\left(\overline{B C}_{11}+\overline{B C}_{22}\right)
$$

$$
\begin{gathered}
=49(2 m+2 i)-64\left(2 m+1 / 4 \mathrm{~h}+\frac{98}{64} i+\frac{1}{32} l\right) \\
=98 m+98 i-128 m-16 h-98 i-2 l \\
=-30 \mathrm{~m}-16 h-2 l
\end{gathered}
$$

When compensated $\mathrm{h}$ and $\mathrm{L}$ from above Equations 1 and 2.

$$
\begin{aligned}
& \mathrm{L}=32 \overline{\mathrm{F}}_{3}-64 \overline{\mathrm{F}}_{4}+32 \mathrm{~m} \\
& \mathrm{~h}=16 \overline{\mathrm{F}}_{4}-4 \overline{\mathrm{F}}_{3}-12 \mathrm{~m}
\end{aligned}
$$

$$
\begin{aligned}
& 49\left(\overline{\mathrm{P}}_{1}+\overline{\mathrm{P}}_{2}\right)-64\left(\overline{B C}_{31}+\overline{B C}_{32}\right)=-30 \mathrm{~m}-16 h-2 l \\
& =-30 \mathrm{~m}-16\left(16 \overline{\mathrm{F}}_{4}-4 \overline{\mathrm{F}}_{3}-12 \mathrm{~m}\right)-2\left(32 \overline{\mathrm{F}}_{3}-64 \overline{\mathrm{F}}_{4}+32 \mathrm{~m}\right) \\
& 49\left(\overline{\mathrm{P}}_{1}+\overline{\mathrm{P}}_{2}\right)-64\left(\overline{B C}_{31}+\overline{B C}_{32}\right)=98 \mathrm{~m}-128 \overline{\mathrm{F}}_{4}
\end{aligned}
$$


Equation for estimating mean effect (M)

$\mathrm{m}=\frac{1}{98}\left(49 \overline{\mathrm{P}}_{1}+49 \overline{\mathrm{P}}_{2}-64 \overline{B C}_{31}-64 \overline{B C}_{32}+128 \overline{\mathrm{F}}_{4}\right)$

When $\mathrm{m}$ is compensated in formula of $\mathrm{L}$ in Equation 2 we can estimate $\mathrm{L}$ as follow

$\mathrm{L}=32 \overline{\mathrm{F}}_{3}-64 \overline{\mathrm{F}}_{4}+\frac{32}{98}\left(49 \overline{\mathrm{P}}_{1}+49 \overline{\mathrm{P}}_{2}-64 \overline{B C}_{31}-64 \overline{B C}_{32}+128 \overline{\mathrm{F}}_{4}\right)$

Formula for estimating Dominance $x$ dominance effect $(L)$ :

$\mathrm{L}=\frac{16}{49}\left(98 \overline{\mathrm{F}}_{3}-68 \overline{\mathrm{F}}_{4}+49 \overline{\mathrm{P}}_{1}+49 \overline{\mathrm{P}}_{2}-64 \overline{B C}_{31}-64 \overline{B C}_{32}\right)$

When $\mathrm{m}$ is compensated in formula of $\mathrm{H}$ in Equation 1 we can estimate $\mathrm{H}$ as follow

$\mathrm{H}=16 \overline{\mathrm{F}}_{4}-4 \overline{\mathrm{F}}_{3}-\frac{12}{98}\left(49 \overline{\mathrm{P}}_{1}+49 \overline{\mathrm{P}}_{2}-64 \overline{B C}_{31}-64 \overline{B C}_{32}+128 \overline{\mathrm{F}}_{4}\right)$

Formula for estimating Dominance effect $(H)$

$\mathrm{h}=\frac{1}{49}\left(384 \overline{B C}_{31}+384 \overline{B C}_{32}-196 \overline{\mathrm{F}}_{3}-294 \overline{\mathrm{P}}_{1}-294 \overline{\mathrm{P}}_{2}+16 \overline{\mathrm{F}}_{4}\right)$

$\mathrm{D}$ is coered as half of the difference between the two parents.

Equation for estimating the additive effect:

$\mathrm{d}=\frac{1}{2}\left(\bar{P}_{1}-\bar{P}_{2}\right)$

For estimating Additive $x$ Additive (i) $\overline{B C}_{31}$ and $\overline{B C}_{32}$ means are summed together and compensated $\mathrm{h}$ and $\mathrm{L}$ from above formula 1 and 2:

$$
\begin{aligned}
& \overline{B C}_{31}+\overline{B C}_{32}=m+7 / 8 d+1 / 6 h+\frac{44}{64} i+\frac{l}{64} j+\frac{1}{64} l+m-7 / 8 d+1 / 6 h+\frac{4 y}{64} i-\frac{1}{64} j+\frac{1}{64} l \\
& \overline{B C}_{31}+\overline{B C}_{32}=2 m+1 / 4 \mathrm{~h}+\frac{98}{64} i+\frac{1}{32} l
\end{aligned}
$$$$
\overline{B C}_{31}+\overline{B C}_{32}=2 m+1 / 4\left(16 \overline{\mathrm{F}}_{4}-4 \overline{\mathrm{F}}_{3}-12 \mathrm{~m}\right)+\frac{98}{64} i+\frac{1}{32}\left(32 \overline{\mathrm{F}}_{3}-64 \overline{\mathrm{F}}_{4}+32 \mathrm{~m}\right)
$$

Equation for estimating the Additive x Additive effect (i).

$$
\mathrm{i}=\frac{32}{49}\left(\overline{B C}_{31}+\overline{B C}_{32}-2 \overline{\mathrm{F}}_{4}\right)
$$

For estimating $\mathrm{j}$ by subtract $\overline{B C}_{31}$ and $\overline{B C}_{32}$ means and compensated $\mathrm{D}$ from above formula

$\overline{B C}_{31}-\overline{B C}_{n 2}=m+7 / 8 d+7 / 8 h+\frac{44}{64} i+\frac{7}{64} j+\frac{1}{64} l-m+7 / 8 d-7 / 8 h-\frac{44}{64} i+\frac{7}{64} j-\frac{1}{64} l$

$$
\begin{aligned}
& \overline{B C}_{31}-\overline{B C}_{32}=\frac{\gamma}{4} d+\frac{\gamma}{32} j \\
& \overline{B C}_{31}-\overline{B C}_{32}=\frac{7}{4} * \frac{1}{2}\left(\overline{\mathrm{P}}_{1}-\overline{\mathrm{P}}_{2}\right)+\frac{\gamma}{32} j
\end{aligned}
$$$$
\mathbf{j}=\frac{32}{56}\left(8 \overline{B C}_{31}-8 \overline{B C}_{32}-7 \overline{\mathrm{P}}_{1}+7 \overline{\mathrm{P}}_{2}\right)
$$

Equation for estimating the Additive $x$ dominance effect $(J)$

$$
\mathrm{j}=\frac{1}{7}\left(32 \overline{B C}_{31}-32 \overline{B C}_{32}-28 \overline{\mathrm{P}}_{1}+28 \overline{\mathrm{P}}_{2}\right)
$$

\section{Algebraic proof}

Algebraic proof of scaling tests formulae

Note that, the following proof depends only on additive-dominance model which means no nonallelic interaction.

$$
\begin{aligned}
A & =4 \bar{F}_{4}-2 \bar{F}_{3}-\bar{P}_{1}-\bar{P}_{2} \\
& =4(\mathrm{~m}+1 / 8 \mathrm{~h})-2(\mathrm{~m}+1 / 4 \mathrm{~h})-(\mathrm{m}+\mathrm{d})-(\mathrm{m}-\mathrm{d}) \\
& =\mathrm{m}(4-2-1-1)+\mathrm{h}(1 / 2-1 / 2)+\mathrm{d}(1-1)=\text { zero }
\end{aligned}
$$




$$
\begin{aligned}
& E=\overline{B C}_{31}+\overline{B C}_{32}-2 \bar{F}_{4} \\
& \quad=(\mathrm{m}+7 / 8 \mathrm{~d}+1 / 8 \mathrm{~h})+(\mathrm{m}-7 / 8 \mathrm{~d}+1 / 8 \mathrm{~h})-2(\mathrm{~m}+ \\
& 1 / 8 \mathrm{~h}) \\
& \quad=\mathrm{m}(1+1-2)+\mathrm{d}(7 / 8-7 / 8)+\mathrm{h}(1 / 8+1 / 8-1 / 4)=\text { zero }
\end{aligned}
$$

Algebraic proof of six parameters $M, D, H, I$, $J$ and $L$

$$
\begin{aligned}
& \mathrm{m}=\frac{1}{98}\left(49 \overline{\mathrm{P}}_{1}+49 \overline{\mathrm{P}}_{2}-64 \overline{B C}_{31}-64 \overline{B C}_{32}+128 \overline{\mathrm{F}}_{4}\right) \\
& =1 /{ }_{98}[49(\mathrm{~m}+\mathrm{d}+\mathrm{i}) \\
& +49(\mathrm{~m}-\mathrm{d}+\mathrm{i}) \\
& +128\left(\mathrm{~m}+1 / 8 \mathrm{~h}+1 /{ }_{64} \mathrm{l}\right) \\
& -64\left(m+7 / 8 d+1 / 8 h+49 / 64+1 /{ }_{64} j+1 / 64\right) \\
& \left.-64\left(m-7 / 8 d+1 / 8 h+{ }^{49} /{ }_{64} i-1 /{ }_{64} j+1 /{ }_{64} l\right)\right] \\
& =\left[1 /{ }_{98}(98)\right] \mathbf{m}=\mathbf{m} \\
& \mathrm{d}=\frac{1}{2}\left(\bar{P}_{1}-\bar{P}_{2}\right) \\
& +1 / 2(m+d+i) \\
& -1 / 2(m-d+i) \\
& =1 / 2 \mathrm{~d}+1 / 2 \mathrm{~d}=\mathbf{d}
\end{aligned}
$$$$
\mathrm{h}=\frac{1}{49}\left(384 \overline{B C}_{31}+384 \overline{B C}_{32}-196 \overline{\mathrm{F}}_{3}-294 \overline{\mathrm{P}}_{1}-294 \overline{\mathrm{P}}_{2}+16 \overline{\mathrm{F}}_{4}\right)
$$$$
=1 /{ }_{49}\left[384\left(\mathrm{~m}+7 / 8 \mathrm{~d}+1 / 8 \mathrm{~h}+{ }^{49} /{ }_{64} \mathrm{i}+1 /{ }_{64} \mathrm{j}+1 /{ }_{64} \mathrm{l}\right)\right.
$$$$
+384\left(\mathrm{~m}-7 / 8 \mathrm{~d}+1 / 8 \mathrm{~h}+{ }^{49} /{ }_{64} \mathrm{i}-1 /{ }_{64} \mathrm{j}+1 /{ }_{64} \mathrm{l}\right)
$$$$
\text { - } 294(m-d+i)
$$$$
\text { - } 294(m+d+i)
$$$$
+16(\mathrm{~m}+1 / 8 \mathrm{~h}+1 / 64)
$$$$
\text { - } 196(\mathrm{~m}+1 / 4 \mathrm{~h}+1 / 16 \mathrm{l})]
$$

49) $\left.1 /{ }_{49}=\mathrm{H}\right)=\mathbf{h}$

$$
\begin{aligned}
\mathrm{i}= & \frac{32}{49}\left(\overline{B C}_{31}+\overline{B C}_{32}-2 \overline{\mathrm{F}}_{4}\right) \\
=32 / 49 & {\left[\left(\mathrm{~m}+7 / 8 \mathrm{~d}+1 / 8 \mathrm{~h}+49 /{ }_{64} \mathrm{i}+7 / 64 \mathrm{j}+1 /{ }_{64} \mathrm{l}\right)\right.} \\
& \quad+\left(\mathrm{m}-7 / 8 \mathrm{~d}+1 / 8 \mathrm{~h}+49 /{ }_{64} \mathrm{i}-7 / 64 \mathrm{j}+1 /{ }_{64} \mathrm{l}\right)
\end{aligned}
$$

$$
\begin{aligned}
& \left.-2\left(\mathrm{~m}+1 / 8 \mathrm{~h}+1 /{ }_{64} 1\right)\right] \\
= & \left(32 / 49 \times{ }_{32}^{49}\right) \mathrm{i}=\mathbf{i} \\
\mathbf{j}= & \frac{\mathbf{1}}{\mathbf{7}}\left(32 \overline{B C}_{31}-32 \overline{B C}_{32}-28 \overline{\mathrm{P}}_{1}+28 \overline{\mathrm{P}}_{2}\right)
\end{aligned}
$$

$$
\begin{aligned}
&=32] \frac{1}{7}\left(\mathrm{~m}+7 / 8 \mathrm{~d}+1 / 8 \mathrm{~h}+49 / 64 \mathrm{i}+7 / 64 \mathrm{j}+1 /{ }_{64} \mathrm{l}\right) \\
&-32\left(\mathrm{~m}-7 / 8 \mathrm{~d}+1 / 8 \mathrm{~h}+49 /{ }_{64} \mathrm{i}-{ }^{7 / 64} \mathrm{j}+1 /{ }_{64} \mathrm{l}\right) \\
&-28(\mathrm{~m}+\mathrm{d}+\mathrm{i}) \\
&+28(\mathrm{~m}-\mathrm{d}+\mathrm{i})] \\
&=\left(1 /{ }_{7}\right)(7 \mathbf{j})=\mathbf{j} \\
& \mathrm{L}=\frac{16}{49}\left(98 \overline{\mathrm{F}}_{3}-68 \overline{\mathrm{F}}_{4}+49 \overline{\mathrm{P}}_{1}+49 \overline{\mathrm{P}}_{2}-64 \overline{B C}_{31}-64 \overline{B C}_{32}\right)
\end{aligned}
$$$$
\text { 49] }{ }^{16 / 49}=(\mathrm{m}+\mathrm{d}+\mathrm{i})
$$$$
+49(\mathrm{~m}-\mathrm{d}+\mathrm{i})
$$$$
-68(m+1 / 8 h+1 / 64)
$$$$
+98\left(\mathrm{~m}+1 /{ }_{4} \mathrm{~h}+1 /{ }_{16} \mathrm{l}\right)
$$$$
-64\left(m+7 / 8 d+1 / 8 h+49 / 64+1 /{ }_{64} j+1 / 64\right)
$$$$
\left.-64\left(m-7 / 8 d+1 / 8 h+49 /{ }_{64} \mathrm{i}-1 /{ }_{64} \mathrm{j}+1 /{ }_{64} \mathrm{l}\right)\right]
$$$$
49 / 16) 16 / 49=\mathrm{L})=\mathbf{L}
$$

Mathematical proof via working example

To validate the new model, real data of mean $(\mathrm{m})$, additive $(\mathrm{d})$, dominance $(\mathrm{h})$, additive $x$ additive (i), additive $x$ dominance $(j)$ and dominance $\mathrm{x}$ dominance (l) gene effects in the six-parameter model for phosphorus-use efficiency in cross number one of soybean were used (Veronica et $a l, 2017$ ) as shown in the following table.

\begin{tabular}{|c|c|c|c|c|c|c|}
\hline $\begin{array}{l}\text { Gen- } \\
\text { otype }\end{array}$ & $\mathrm{m}$ & $\mathrm{d}$ & $\mathrm{h}$ & $\mathrm{i}$ & $\mathrm{j}$ & $\mathrm{l}$ \\
\hline Value & 0.02 & 0.01 & -0.02 & 0.003 & -0.01 & 0.01 \\
\hline
\end{tabular}

Thus, the expected genetic means of the different population deduced according to their genotypic segregation distribution would be as follows: 


$$
\begin{aligned}
& \overline{\mathrm{P}}_{1}=\mathrm{m}+\mathrm{d}+\mathrm{i}=0.02+0.01+0.003=0.033 \\
& \overline{\mathrm{P}}_{2}=\mathrm{m}-\mathrm{d}+\mathrm{i}=0.02-0.01+0.003=0.013 \\
& \overline{\mathrm{F}}_{3}=\mathrm{m}+1 / 4 \mathrm{~h}+\frac{1}{16} \mathrm{l}=0.02+1 / 4(-0.02)+\frac{1}{16}(0.01)=0.015625 \\
& \overline{\mathrm{F}}_{4}=\mathrm{m}+1 / 8 \mathrm{~h}+\frac{1}{64} l=0.02+1 / 8(-0.02)+\frac{1}{64}(0.01)=0.017656 \\
& \left.\overline{B C}_{31}=m+7 / 8 d+48 h+\frac{49}{64} i+\frac{7}{64} j+\frac{1}{64}\right] \\
& =0.02+78(0.01)+48(-0.02)+\frac{49}{64}(0.003)+\frac{7}{64}(-0.01)+\frac{1}{64}(0.01)=0.027609
\end{aligned}
$$$$
\overline{B C}_{32}=m-7 / 8 d+48 n+\frac{49}{64}-\frac{7}{64} i+\frac{1}{64}
$$$$
=0.02-7 / 8(0.01)+48(-0.02)+\frac{49}{64}(-0.01)-\frac{7}{64}(-0.01)+\frac{1}{64}(0.01)=0.012227
$$

\begin{tabular}{|l|c|c|c|c|c|c|}
\hline Populations & $\overline{\mathbf{P}}_{\mathbf{1}}$ & $\overline{\mathbf{P}}_{\mathbf{2}}$ & $\overline{\mathbf{F}}_{\mathbf{3}}$ & $\overline{\mathbf{F}}_{4}$ & $\overline{\mathbf{B C}}_{31}$ & $\overline{\mathbf{B C}}_{32}$ \\
\hline $\begin{array}{l}\text { Genetic } \\
\text { Mean }\end{array}$ & 0.033 & 0.013 & 0.015625 & 0.017656 & 0.027609 & 0.012297 \\
\hline
\end{tabular}

Re-estimation of population genetic means based on the proposed formulae

$\mathrm{m}=\frac{1}{98}\left(49 \overline{\mathrm{P}}_{1}+49 \overline{\mathrm{P}}_{2}-64 \overline{B C}_{31}-64 \overline{B C}_{32}+128 \overline{\mathrm{F}}_{4}\right)=0.02$ $\mathrm{m}=\frac{1}{98}(49 \times 0.033+49 \times 0.013-64 \times 0.027609-64 \times 0.0122297+1.28 \times 0.017656)=0.02$

$$
\begin{aligned}
& \mathbf{d}=\frac{1}{2}\left(\bar{P}_{1}-\bar{P}_{2}\right) \\
& \mathbf{d}=\frac{1}{2}(0.033-0.013)=0.01
\end{aligned}
$$

$\mathrm{h}=\frac{1}{49}\left(384 \overline{B C}_{n 1}+384 \overline{B C}_{n 2}-196 \overline{\mathrm{F}}_{9}-294 \overline{\mathrm{P}}_{1}-294 \overline{\mathrm{P}}_{2}+16 \overline{\mathrm{P}}_{4}\right)=-0,02$

$h=\frac{1}{49}(384 \times 0.0276009+384 \times 0.0122297-1.96 \times 0.0155025-294 \times 0.033-204+0.013+16$ $\times 0.017656)=-0,02$

$\mathrm{i}=\frac{32}{49}\left(\overline{B C}_{31}+\overline{B C}_{32}-2 \overline{\mathrm{F}}_{4}\right)=0.003$

$\mathrm{i}=\frac{32}{49}(0.027609+0.012297-2 * * 0.017656)=0.003$

$\mathfrak{j}=\frac{1}{7}\left(32 \overline{B C}_{31}-32 \overline{B C}_{32}-28 \overline{\mathrm{P}}_{1}+28 \overline{\mathrm{P}}_{2}\right)=-0.01$ $j=\frac{1}{7}(32 * 0.027609-32 * 0.012297-28 * 0.033+28 * 0.013)=-0.01$

$\mathrm{L}=\frac{10}{49}\left(98 \overline{\mathrm{F}}_{3}-68 \overline{\mathrm{F}}_{4}+49 \overline{\mathrm{P}}_{1}+49 \overline{\mathrm{P}}_{2}-64 \overline{B C}_{31}-64 \overline{B C}_{32}\right)=0.01$

$L=\frac{16}{49}(98 \times 0.015625-68 \times 0.017656+49 \times 0.033+49 \times 0.013-64 \times 0.027609-64 \times 0.012229)$ $=0,01$

\section{RESULTS}

The proposed model in this paper is similar to that model developed by Mather \& Jinks (1982) for estimating all types of genetic effects. However, algebraic and mathematical proofs revealed that the proposed model can be applied to estimate all types of genetic effects with different means of advanced populations of $\bar{P}_{1}, \bar{P}_{2}, \bar{F}_{3}, \bar{F}_{4}, \overline{B C}_{31}$ and $\overline{B C}_{32}$ - The results showed that, if epistasis is absent according to scaling test (A, B, C, $\mathrm{D}$ and $\mathrm{E})$ the following equations are used: $\operatorname{Mean}(\mathrm{m})=\frac{1}{4}\left(49 \overline{\mathrm{P}}_{1}+49 \overline{\mathrm{P}}_{2}-64 \overline{B C}_{n 1}-64 \overline{B C}_{n}+128 \overline{\mathrm{F}}_{4}\right)$

Additive effect $(\mathbf{d})=\frac{1}{2}\left(\bar{P}_{1}-\bar{P}_{2}\right)$ and Dominance effect $(\mathrm{h})=\frac{1}{49}\left(384 \overline{B C}_{71}+384 \overline{B C}_{32}-196 \overline{\mathrm{F}}_{9}-294 \overline{\mathrm{P}}_{1}-294 \overline{\mathrm{P}}_{2}+16 \overline{\mathrm{F}}_{4}\right)$

However, if epistasis is present, the following equations are used:

Additive $*$ Addtive eff ect $(i)=\frac{32}{49}\left(\overline{B C}_{31}+\overline{B C}_{32}-2 \overline{\mathrm{F}}_{4}\right)$,

Additive $*$ Dominance effect $(\mathrm{j})=\frac{1}{7}\left(32 \overline{B C}_{31}-32 \overline{B C}_{32}-28 \overline{\mathrm{P}}_{1}+28 \overline{\mathrm{P}}_{2}\right)$ and

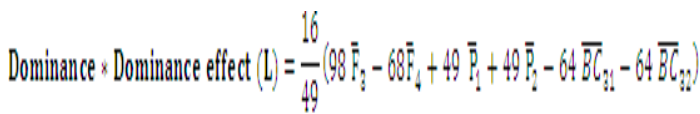

Finally, the real data analysis in soybean (Veronica et al, 2017) as an example to validate the equations, suggest that the model can serve as a viable particularly to estimate epistasis, additive and dominance genetic effects using the data generated from advanced filial and backcross populations $\overline{\boldsymbol{P}}_{1}, \overline{\boldsymbol{P}}_{2}, \overline{\boldsymbol{F}}_{3}, \overline{\boldsymbol{F}}_{4}, \overline{\boldsymbol{B C}}_{31}$ and $\overline{\boldsymbol{B C}}_{32}$.

Egypt.J.Agron. Vol.39, No.1(2017) 


\section{CONCLUSION}

In this work, an algebraic solution, algebraic and mathematical proof via real data example were used to develop six equations for estimating six types of gene effects as a novel method. The proposed model can utilize the advanced populations for estimating the genetic effects. Thus, the model can help the breeders especially those working with advanced populations of filial and backcross to estimate the genetic effects with simple equations.

\section{REFERENCES}

Anderson,V.L. and Kempthorne, O. (1954) A model for the study of quantitative inheritance. Genetics,39, 883- 898 .

Carlborg, Ö., Hocking, P. M., Burt, D. W. and Haley, C. S. (2004) Simultaneous mapping of epistatic QTL in chickens reveals clusters of QTL pairs with similar genetic effects on growth. Genet. Res. 83, 197-209.

Cavalli, L.L.(1952) An analysis of linkage in quantitative inheritance in "Quantitative Inheritance" E.C.R. Reeve and C.H. Waddington (Ed.), pp. 135-144. HMSO London..

Chahal, G.S.and Jink J.L. (1978) A general method of detecting the additive, dominance and epistatic variation that inbred lines can generate using a single tester. Heredity, 40, 117-125.

Checa, O., Ceballos, H. and Blair, M. W. (2006) Generation means analysis of climbing ability in common bean (Phaseolus vulgaris L.). J. Hered. 97, 456-465.

Cockerham, C.C.(1954) An extension of the concept of partitioning hereditary variance for analysis of covariances among relatives when epistasis is present. Genetics, 39,859-882.

Gardner, C.O. and Eberhart, S.A., (1966) Analysis and interpretation of variety cross diallel and related population. Biometrics, 22, 439-452.

Hayman, B.I., (1957) Interaction, heterosis and diallel crosses. Genetics, 42, 336-355.

Hayman, B.I. (1958) The separation of epistatic from additive and dominance variations in generation means. Heredity, 12, 371-390

Jinks, J.L. (1956) The F2 and backcross generations from a set of diallel crosses. Heredity, 10,1-30.

Jinks, J.L., Perkins, J.M. and Breese, E.L. (1969) A general method of detecting additive, dominance and epistatic variation for metric traits. II. Application to inbred line. Heredity, 24, 45-57.

Jinks, J.L. and Jones, R.M. (1958) Estimation of the components of heterosis. Genetics, 43, 223-234.

Kearsey, M.J. and Jinks, J.L.(1968) Ageneral method of detecting additive, dominance and epistatic variation for metrical traits I. Theory. Heredity, 23, 403-409.

Iqbal, M. Z., and Nadeem, M. A. (2003) Generation mean analysis for seed cotton yield and number of sympodial branches per plant in cotton (Gossypium hirsutum L.). Asian J. Plant Sci. 2, 395-399.

Mather, K.(1949) Biometrical Genetics."Methuen and Co. Ltd., London.

Mather, K. and Jinks, J.L. (1971) Biometrical Genetics."Chapman and Hall, London.

Mather, K. and Jinks, I.L. (1982) Biometrical Genetics" $3^{\text {rd }}$ ed. Champan and Hall, London.

Pooni, H. S. and Treharne, A. J. (1994) The role of epistasis and background genotype in the expression of heterosis. Heredity, 72, 628-635.

Sharmila, V., Ganesh, S. K. and Gunasekaran, M. (2007) Generation mean analysis for quantitative traits in sesame (Sesamum indicum L.) crosses. Gen. Mol. Biol. 30, 80-84.

Veronica, N. E. Uzokwe, B. A., I. F., Robert, A., Inakwu, O. A. O., David, K. O., Kenton, D. and Nteranya, S., (2017) Generation mean analysis of phosphorus-use efficiency in freely nodulating soybean crosses grown in low-phosphorus soil. Plant Breeding 136, Early View (Online Version of Record published 


\section{نموذج وراثى احصائى لتقدير التاثيرات الوراثية الاضافية والسيادية والتفوق باستخدام عثائر

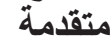 \\ احمد السيد احمد خلف، توفيق محمد ثابت" واحمد عبدالفتاح يسنة

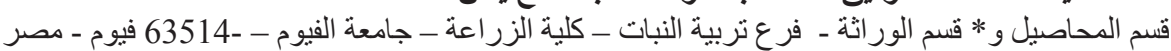

العديد من الدراسات تناولت تقدير التأثير ات الور اثية التى تتحكم فى الصفات الكمية. معظم طرق التقدير تركز على منوسطات الاجيال المبكرة مثل فئ

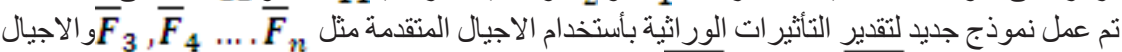
الرجعية المتقدمة2 $\overline{B C}_{32}, \overline{B C}_{31,} \bar{F}_{4}, \bar{P}_{2}, \bar{F}_{3}, \bar{P}_{1}$ لمتوسطات اجيال عشائر كلا

$$
\begin{aligned}
& \frac{1}{98}\left(49 \overline{\mathrm{P}}_{1}+49 \overline{\mathrm{P}}_{2}-64 \overline{\mathrm{BC}}_{31}-64 \overline{\mathrm{BC}}_{32}+128 \overline{\mathrm{F}}_{4}\right)=\mathrm{M} \text { المتول } \\
& \frac{1}{2}\left(\overline{\mathrm{P}}_{1}-\overline{\mathrm{P}}_{2}\right)=(\mathrm{D}) \text { (الثاثير الاضافي } \\
& \text { التأثير السيادى (H) } \\
& \frac{1}{49}\left(384 \overline{\mathrm{BC}}_{31}+384 \overline{\mathrm{BC}}_{32}-196 \overline{\mathrm{F}}_{3}-294 \overline{\mathrm{P}}_{1}-294 \overline{\mathrm{P}}_{2}+16 \overline{\mathrm{F}}_{4}\right)
\end{aligned}
$$

$$
\text { وثناثة انواع من التفوق }
$$

$$
\begin{aligned}
& \frac{32}{49}\left(\overline{\mathrm{BC}}_{31}+\overline{\mathrm{BC}}_{32}-2 \overline{\mathrm{F}}_{4}\right)=(\mathrm{I}) \text { الأثير الاضافى X الاضافي }
\end{aligned}
$$

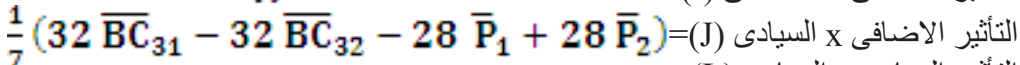

$$
\begin{aligned}
& \text { التأثير السيادى X السيادى X) الإنيادى (J) } \\
& \frac{16}{49}\left(98 \overline{\mathrm{F}}_{3}-68 \overline{\mathrm{F}}_{4}+49 \overline{\mathrm{P}}_{1}+49 \overline{\mathrm{P}}_{2}-64 \overline{\mathrm{BC}}_{31}-64 \overline{\mathrm{BC}}_{32}\right)
\end{aligned}
$$

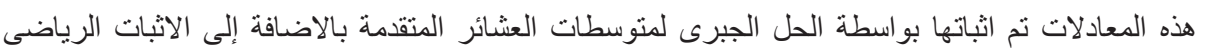

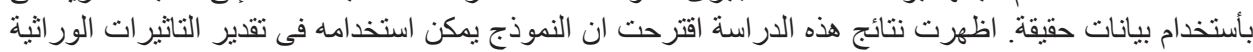

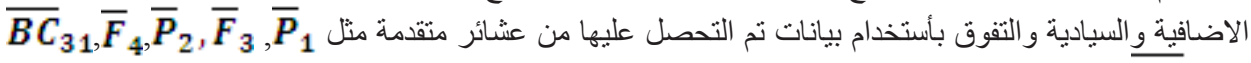
$\overline{B C}_{32}$, 